\title{
Comparative efficacy of Chinese herbal injections for treating chronic heart failure: a network meta-analysis
}

\author{
Kai-Huan Wang, Jia-Rui Wu* (D), Dan Zhang, Xiao-Jiao Duan and Meng-Wei Ni
}

\begin{abstract}
Background: On account of deterioration of chronic heart failure (CHF) and extensive exploration of Chinese herbal injections (CHIs), we performed a network meta-analysis to investigate the efficacy of CHls (Huangqi injection, Shenfu injection, Shengmai injection, Shenmai injection, Shenqi Fuzheng injection, Yiqifumai injection) on the basis of western medicine (WM) treatment in CHF.

Methods: Literature search was conducted in Embase, the Cochrane Library, Pubmed, Chinese Biological Medicine Database, China National Knowledge Infrastructure, Wanfang Database, Chinese Scientific Journal Database from inception to June 12nd 2017, and study selection was abided by a prior eligible criteria.

Results: Ultimately, a total of 113 randomized controlled trials (RCTs) were enrolled. The clinical data of the effective clinical rate, left ventricular ejection fraction, cardiac output and others outcomes was estimated by Stata software and Winbugs software. Risk of bias was assessed by Cochrane Collaboration's tools. Integrating the each outcome's results, a combination of Shengmai injection/Shenmai injection and WM obtain a first rank in most outcomes, particularly primary outcomes.
\end{abstract}

Conclusions: In conclusion, on the basis of WM, Shengmai injection or Shenmai injection may be a perforable treatment in CHF. In terms of insufficient of this study, more high quality RCTs needed to implement to support our conclusions.

Keywords: Network meta-analysis (NMA), Chronic heart failure (CHF), Chinese herbal injection (CHI)

\section{Background}

Chronic heart failure (CHF) refers to a pathologic condition that cardiac output is absolute or relative reduce and cannot meet the whole body tissue metabolism under the normal venous return, then result in decreasing the myocardial contractile force and ventricular compliance, ultimately dyspnea, edema, feeble and so on. It was estimated that five-year survival rate of $\mathrm{CHF}$ was lower as malignant tumor and $\mathrm{CHF}$ was a main reason of disability and death on a global scale [1-3]. Impaired cardiac function of CHF patients may lessen their ability of daily living and render them a heavy economic pressure $[1,4]$. At present, the primary aims of alleviating CHF symptoms are to inhibit myocardial

\footnotetext{
*Correspondence: exogamy@163.com

Department of Clinical Pharmacology of Traditional Chinese Medicine, School of Chinese Materia Medica, Beijing University of Chinese Medicine, Beijing 100102, China
}

(c) The Author(s). 2018 Open Access This article is distributed under the terms of the Creative Commons Attribution 4.0 International License (http://creativecommons.org/licenses/by/4.0/), which permits unrestricted use, distribution, and reproduction in any medium, provided you give appropriate credit to the original author(s) and the source, provide a link to the Creative Commons license, and indicate if changes were made. The Creative Commons Public Domain Dedication waiver (http://creativecommons.org/publicdomain/zero/1.0/) applies to the data made available in this article, unless otherwise stated. angiotensin-converting enzyme inhibitors (ACEIs), angiotensin II receptor blockers (ARBs), digoxin, and diuretics are become standard western medicine (WM) treatment in CHF [6], while it cannot obtain a desired effect own to poor compliance, lower heart rate of patients and others questions [5]. In consideration of its limitations, the application of Chinese herbal injections (CHIs) could be promoted. Currently, a combination between CHIs and WM treatment has already been a supportive measure in treatment of $\mathrm{CHF}$ in China. In accordance with traditional Chinese medicine (TCM) theories, CHF pertain to "heart impediment (xin bi)", "palpitation", "edema" and so forth, which caused by heart and then affect others organs. The clinical principle is to strengthen the body resistance to eliminate pathogenic factors [2]. Due to the relative low recognition of CHIs in CHF, this study selected six CHIs 
commonly used in CHF treatment, all of them were authorized by China Food and Drug Administration (CFDA), namely Huangqi injection (HQI), Shenfu injection (SFI), Shengmai injection (SI), Shenmai injection (SMI), Shenqi Fuzheng injection (SQFZI), Yiqifumai injection (YQFMI), to explore and rank their efficacy in CHF by the approach of network meta-analysis (NMA). Compared with conventional pairwise meta-analysis, NMA can sort the interventions via indirect comparison [7]. At the same time, the clinical trials compared those six CHIs head to head was lack. Thus, an attempt to conduct a NMA was necessarily. The goal of this study was to provide evidence-based hierarchies of the comparative efficacy and more insights for selection of CHF treatment.

\section{Methods}

The study was congrunt with The Prisma Extension Statement for Reporting of Systematic Reviews Incorporating Network Meta-analyses of Health Care Interventions [8]. And the Prisma check list was displayed in Additional file 1.

\section{Eligibility criteria and study selection}

A study was considered eligible if it suited for these criteria: 1) randomized controlled trial (RCT); 2) patients enrolled were diagnosed as CHF according to "Guidelines on the Diagnosis and Treatment of Heart Failure" conducted by The Chinese medical association cardiovascular epidemiology branch in 2014 [9] or "Clinical Guideline of New Drugs for Traditional Chinese Medicine" released by CFDA in 2002 [10]. Both of them contained both western diagnostics, the latter included TCM diagnostics as well; 3) patients receive WM treatment (e.g. cardiotonic, diuretic, ACEIs, $\beta$-blocker and so forth), meanwhile patients needed relevant therapy if they had complications during therapeutic process. On the basis of it, the treatment group received one of the included CHIs, the control group received another or just adopted WM. Besides, the dosages of CHIs were reported; 4) RCTs tested the clinical effective rate. The clinical effective rate calculated by this formula: (number of remarkable recovery patients + number of basic recovery patients) / total number of patients * $100 \%$. Cardiac function classification was conformed to the standard issued by New York Heart Association (NYHA) in the United States. Clinical symptoms disappeared and cardiac function improved 2 levels at least was deemed as the class of remarkable recovery, clinical symptoms relieved and cardiac function increase 1 level was classified into the part of basic recovery, clinical symptoms and cardiac function was unaltered or worse belonged to deterioration. Besides, the incidence of left ventricular ejection fraction (LVEF), cardiac output (CO), stroke volume (SV), 6-min walk test (6MWT), brain natriuretic peptide (BNP), left ventricular end-diastolic dimension (LVEDD), left ventricular end- systolic dimension (LVESD), adverse drug reactions/adverse drug events (ADRs/ADEs) were also evaluated. The clinical effective rate and LVEF were regarded as dominating outcomes of the study, because the clinical effective rate can inflect the efficacy directly and LVEF was a main indicator for CHF. And others were counted as secondary outcomes. A study was excluded when it met these following criteria: 1) the study without full text; 2) duplicated reports; 3) RCTs with incomplete or inaccurate data; 4) RCTs with wrong sequence generation method. For example, sequence generated by odd or even date of birth, some rules based on date (or day) of admission and so forth; 5) patients received physiotherapy, acupuncture and moxibustion therapy, and Chinese materia medica preparation.

A comprehensive literatures searching was carried out in seven database including Embase, the Cochrane Library, Pubmed, Chinese Biological Medicine Database (CBM), China National Knowledge Infrastructure (CNKI), Wanfang Database, Chinese Scientific Journal Database (VIP) from their inception up to June 12nd 2017. In addition, there was no restriction on language. The method that incorporated the medical subject headings $(\mathrm{MeSH})$ term and the free text was applied in searching process, and it would vary from different databases. Each searching item included three parts of terms that chronic heart failure, CHIs, and randomization. Detailed searching strategies were illustrated in Additional file 2 .

After literatures duplicate checking, the rest literatures were firstly screened by titles and abstracts, reviews, irrelevant literatures and animals' experiments reports were excluded. Literatures passed the initial filtration were read full text in order to sort out the eligible RCTs. Two reviewers undertook literature selection respectively, any divergences resolved by discussion or the third reviewer.

\section{Data extraction and quality assessment}

Information from the eligible RCTs was extracted based on a custom-made form. The data consisted of the following items: 1) basic information of the eligibility: the first author, nationality, publication year, study desgin; 2) basic characteristics of patients: sample size, gender composition, average age, course of disease, primary diseases, cardiac function classification; 3) detail of RCTs' intervention; 4) outcomes results and RCTs' quality assessment.

The quality analysis was assessed with the Cochrane Collaboration's tools (version 5.1.0 the Nordic Chchrane Center, the Cochrane Collaboration, 2012 Copenhagen, Denmark) by two reviewers independently. The tool comprised following these 7 items: 1) the method of randomization; 2) the concealment of random allocation; 
3) the blinding method for patients and clinicians; 4) the blinding method for assessor; 5) the integrality of outcomes data; 6) the condition of selective reporting; 7) others bias. Each item was rated as "high risk", "low risk" and "unclear". And any difference between two reviewers settled by discussion or the third reviewer.

It is not necessary for this meta-analysis to obtain an ethical approval, because this study was the procedure that just gathered the clinical data in each RCT without any leak of patients' information.

\section{Statistical analysis}

NMA was performed with Stata software (version 12, Stata Corporation, College Station, Texas, U.S.) and Winbugs (version 1.4, MRC Biostatistics Unit, Cambridge, UK) software by using Mantel-Haenszel random-effects model. In Winbugs software, the number of iteration was set as 50,000, the first 20,000 was used for annealing algorithm in order to eliminate the impact of initial value. For binary outcomes, the pooled results were calculated as odds ratios (ORs). For continuous outcomes, mean differences (MD) were used. Both types of outcomes were presented with their 95\% credible intervals (95\% CIs) as well. Besides, the network graph showed indirect comparative relationship between different interventions was described. The node area of each intervention on behalf of its number of patients, and the thickness between different interventions represented the number of relative RCTs [11]. To rank various CHIs in treatment in CHF, the surface under the cumulative ranking curve (SUCRA) was utilized, which expressed each intervention's efficacy with percentages. A larger area of SCUAR indicated that corresponding intervention was more preferable in certain outcomes [12]. After that, the funnel plots were depicted to reflect publication bias. Due to non-close loops in this NMA, the assumption of consistency between direct and indirect evidence was not utilized.

\section{Results}

\section{Literature selection}

A total of 9968 literatures were identified in initial search (Fig. 1). After removing duplicates, there were 4852 remained. By screened titles and abstracts, 1491 literatures were excluded because they were irrelevant literatures, reviews and animals' experiments reports. 3361 literatures were eligible and then examined respectively, among which 3248 were further excluded, for the following reasons: 1) the RCT's intervention or diseases missed eligibility criteria $(n=2694) ; 2)$ the therapeutic effect standard missed eligibility criteria $(n=256) ; 3)$ the RCT with wrong randomization $(n=68) ; 4)$ the RCT did not divide patients in two groups $(\mathrm{n}=68) ; 5)$ case reports $(n=40) ; 6)$ the RCT without full-text $(n=104) ; 7)$

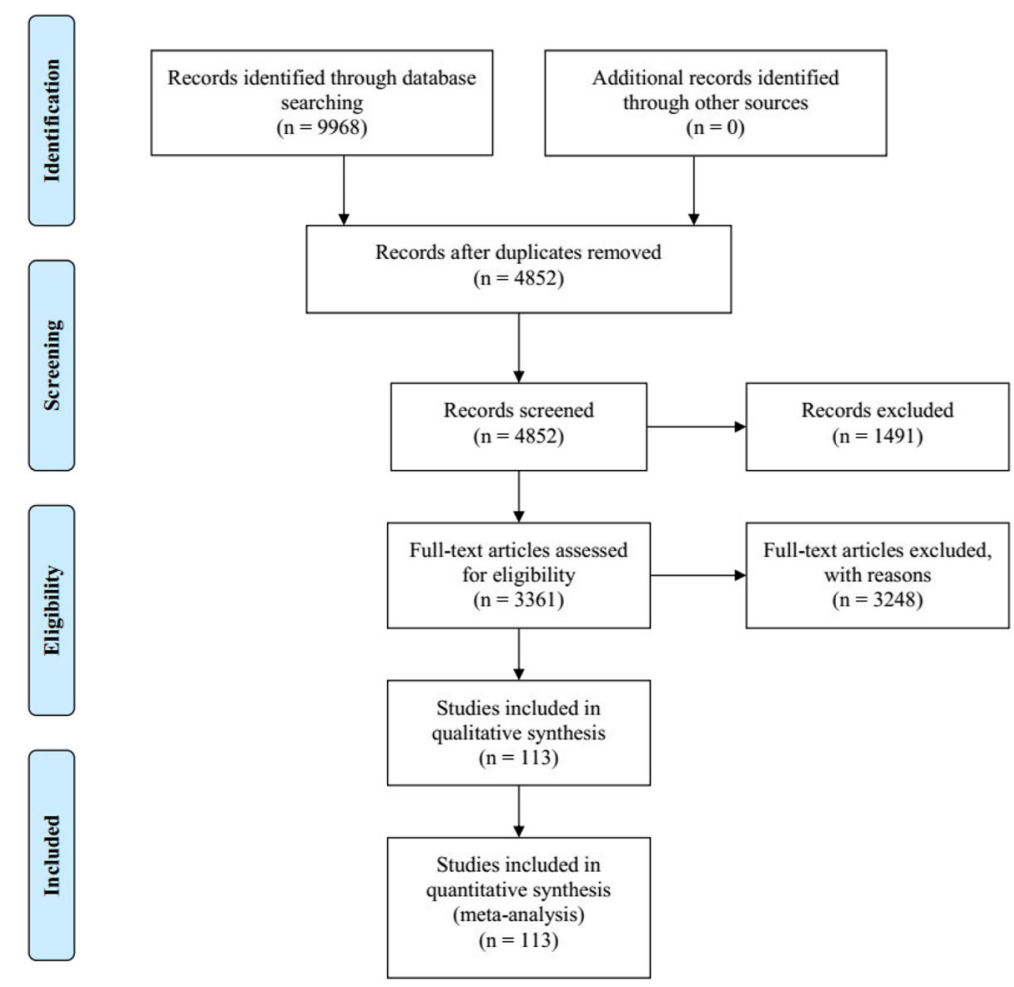

Fig. 1 Flow chart of the search for eligible RCTs 
the RCT with duplicated data $(n=18)$. As results, 113 RCTs that evaluated CHIs combined with WM for CHF were eligible in the NMA, and all of then carried out in China between 2001 and 2017. Meanwhile, 6 types of CHIs were identified, including HQI (12 RCTs), SFI (39 RCTs), SI (31 RCTs), SMI (13 RCTs), SQFZI (12 RCTs) and YQFMI (6 RCTs).

\section{Study characteristics and quality evaluation}

One hundered thirteen [3, 13-124] RCTs with 9525 patients were accorded with the eligible criteria, among which 4852 patients in the treatment groups and 4673 patients in the control groups. Among patients, the male patients were about $55 \%$ of total, and majority of patients were middle aged and elderly people. The intervention of the control groups were WM treatment, for instance, ACEIs, $\beta$-blocker, cardiotonic, diuretic. In the meantime, the treatment groups received one of the identified CHIs on the basis of the control groups. HQI, SFI, SI, SMI was a kind of injection that clinicians injected them with $5 \%-10 \%$ dextrose solution or $0.9 \%$ normal saline, the specific dosage were determined by clinicians. SQFZI was a kind of already made injection with menstruum. And YQFMI was a powder-injection, clinicians injected them with $5 \%-10 \%$ dextrose solution or $0.9 \%$ normal saline as well. All of identified CHIs were injected once a day via mainline. Characteristics of included RCTs can be found in Additional file 3. And the compared connections among each intervention for each outcome were displayed in Fig. 2.

For the eligible RCTs, 19 RCTs [15, 26, 32, 34, 37, 56, $57,61,64,75,81,88,96,101,103,104,107,110,118]$ used the random number table method or sortation randomization method to generate groups and $1 \mathrm{RCTs}$ [88] utilized double blind method. Thus all of them were assessed as low risk. The rest RCTs were evaluated as high risk due to insufficient information. Besides, none of the included RCTs assessed had incomplete data, so the attrition bias was appraised as low risk. As for the part of reporting bias and others bias, the included RCTs
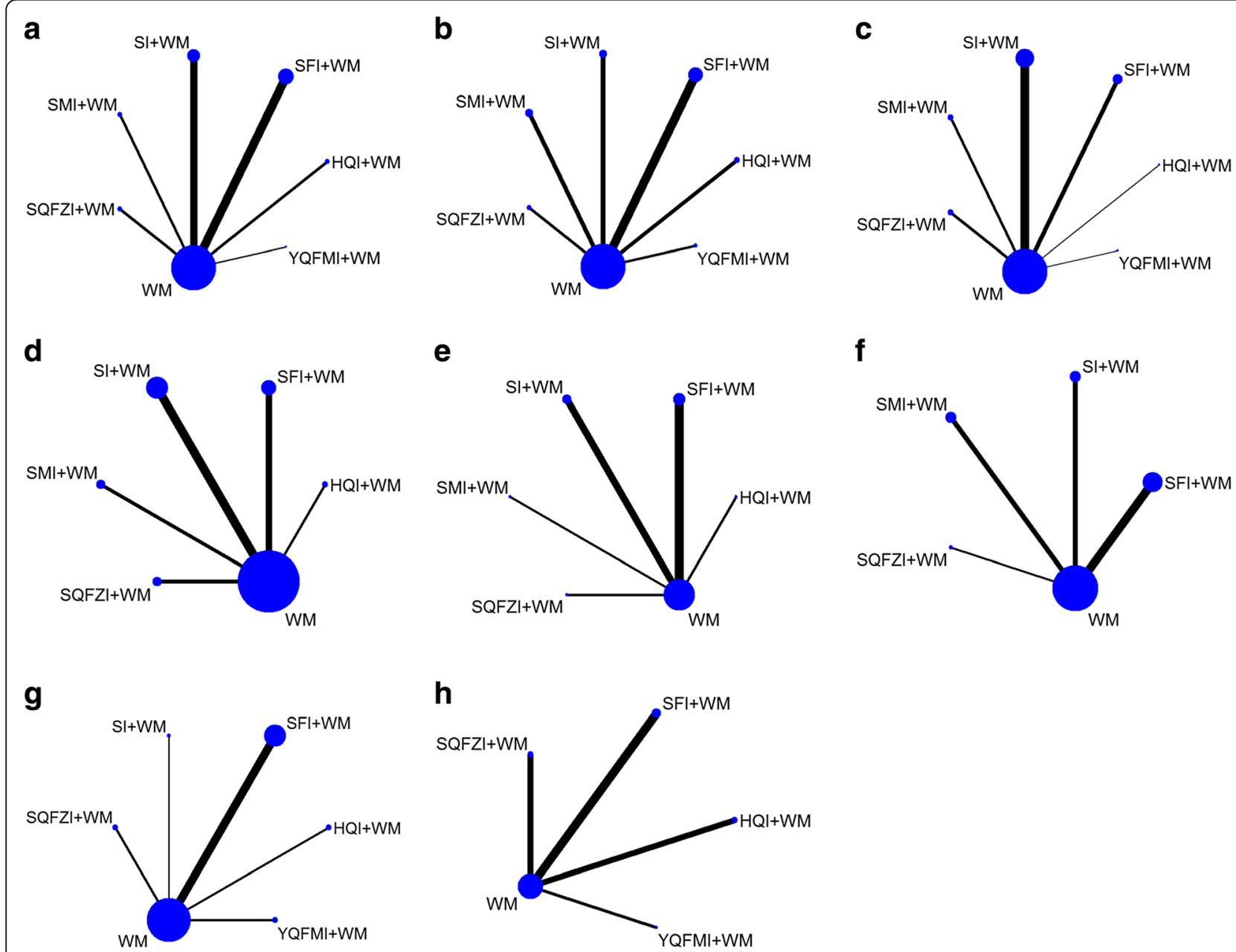

Fig. 2 Network graph of the clinical effective rate, LVEF, CO, SV, 6MWT, BNP, LVEDD and LVESD. Note: a: the clinical effective rate; b: LVEF; c: CO; d: SV; e: 6MWT; f: BNP; $\mathbf{g : ~ L V E D D ; ~} \mathbf{h}$ : LVESD 
did not provide relevant contents about selective report and mention any factors leading to high risk. Therefore these two items were evaluated as unclear risk. The graphical summary was depicted in Fig. 3.

\section{Outcomes}

\section{The clinical effective rate}

The clinical effective rate was deemed as the primary outcomes, as shown in the right upper part of Table 1 $[3,13-124], \quad \mathrm{HQI}+\mathrm{WM} \quad(\mathrm{OR}=0.28, \quad 95 \%$ CIs: $0.19-$ $0.41), \mathrm{SFI}+\mathrm{WM}(\mathrm{OR}=0.29,95 \%$ CIs: 0.24-0.35), SI + WM $(\mathrm{OR}=0.28,95 \%$ CIs: $0.22-0.35), \mathrm{SMI}+\mathrm{WM}(\mathrm{OR}=0.25$, 95\% CIs: $0.17-0.36)$, SQFZI+WM $(\mathrm{OR}=0.28,95 \%$ CIs: 0.19-0.39), YQFMI+WM (OR = 0.42, 95\% CIs: 0.25-0.70), these six interventions with $95 \%$ CIs between 0 and 1 possessed the obvious strengthen in increasing clinical effective rate.

In the Table 5 and Fig. 4, ranking analysis suggested that SMI + WM was the optimal combination, SI + WM was the second and the third was SQFZI+WM.

\section{LVEF}

As the other dominating outcomes, LVEF (\%) was estimated in 57 RCTs $[3,13-15,17,19,20,23,24,32,33$, $36,38,39,42,45-49,53,55,56,58,60,61,64,65,72$, 75, 82, 84, 88, 91-93, 95-97, 99-103, 105, 106, 109-111, 114, 116, 117, 119-123]. According to Table 1, if the 95\% CIs was more than 0 , the result was significant. Four of them were noticeably better than WM treatment for LVEF, as SFI + WM (MD $=4.05,95 \%$ CIs: $1.00-7.59)$, SI + WM (MD = 8.61, 95\% CIs: 4.22-10.99), SMI + WM $(\mathrm{MD}=7.29,95 \%$ CIs: $1.97-12.70)$, YQFMI+WM (MD = 7.26, 95\% CIs: 0.42-13.64) were outstanding among them compared with WM.

Results of ranking analysis manifested that $\mathrm{SI}+\mathrm{WM}$ was efficacious in LVEF. Another beneficial treatments were SMI + WM and YQFMI+WM (Table 5 and Fig. 4).

\section{Co}

CO (L/min) was tested in 22 RCTs [20, 30, 31, 34, 45, $61,68,73,75,76,81-83,87,92,97,99,102,111,112$,
$117,119]$ involved seven interventions. Based on Table 2, only SI + WM (MD $=1.29$, 95\% CIs: $0.74-1.72)$ had excellent performance in improving $\mathrm{CO}$.

The SUCRA mentioned above was also affirmed, SI + WM was the best choice, and the following two were SMI + WM, and SQFZI+WM (Table 5 and Fig. 4).

\section{SV}

SV (ml) was reported in 20 RCTs [20, 23, 30, 36, 38, 45, $61,68,73,75,81,82,87,92,97,99,102,111,112,117]$ involved six interventions. In terms of Table 2 , only SI + WM (MD = 9.35, 95\% CIs: 3.75-14.90) was remarkable among them.

Base on its SUCRA, SI + WM was the optimum, SQFZI+WM was the second and SMI + WM was the third (Table 5 and Fig. 4).

\section{MWT}

The potency of lengthening the distance of 6MWT (m) was assessed, and six interventions with 10 RCTs [24, $32,39,46,51,74,85,92,106,109]$ had data in contrast with WM, shown in Table 3. While the results showed no significant difference in most cases.

The ranking analysis indicated that SI + WM was the favorable intervention (Table 5).

\section{$B N P$}

In terms of BNP $(\mathrm{pg} / \mathrm{ml})$, five treatments with 21 RCTs $[3,33,36,38,44,46,47,53,58,64,65,69,72$, $88,94-97,104,107,109]$ were compared with WM in Table 3. SFI + WM vs SMI + WM $(\mathrm{MD}=80.17,95 \%$ CIs: $16.67-147.5), \quad S F I+W M$ vs SQFZI+WM $(M D=$ 110.00, 95\% CIs: 35.08-186.40), SFI + WM vs WM $(\mathrm{MD}=87.77,95 \%$ CIs: 32.61-129.90) had statistically significance.

Based on ranking analysis, SQFZI+WM attained the first-rank (Table 5).

\section{LVEDD \& LVESD}

The efficiency of decreasing LVEDD (mm) and LVESD $(\mathrm{mm})$ was estimated as well. These two indexes were

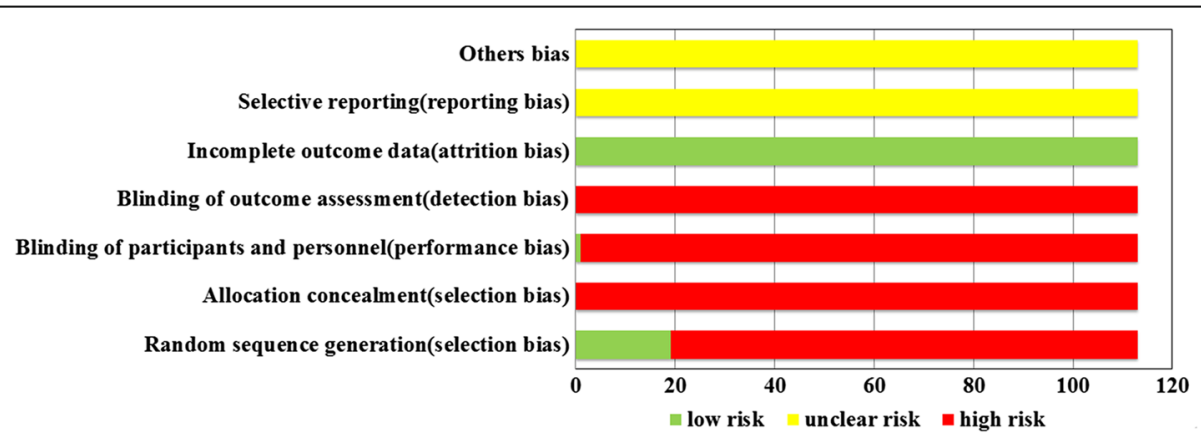

Fig. 3 Risk of bias graph 
Table 1 Odds ratios/mean difference $(95 \% \mathrm{Cls})$ of the clinical effective rate (right upper part) and LVEF (left lower part)

\begin{tabular}{|c|c|c|c|c|c|c|c|}
\hline \multicolumn{8}{|c|}{ the clinical effective rate } \\
\hline \multirow[t]{7}{*}{ LVEF } & $\mathrm{HQI}+\mathrm{WM}$ & $0.98(0.62,1.49)$ & $1.03(0.62,1.59)$ & $1.15(0.65,1.96)$ & $1.00(0.60,1.76)$ & $0.67(0.36,1.28)$ & $\underline{0.28(0.19,0.41)}$ \\
\hline & $0.13(-7.46,7.87)$ & $\mathrm{SFI}+\mathrm{WM}$ & $1.05(0.76,1.41)$ & $1.17(0.79,1.83)$ & $1.05(0.72,1.60)$ & $0.69(0.42,1.21)$ & $4,0.35$ \\
\hline & $-4.31(-11.77,4.08)$ & $-4.50(-8.43,1.48)$ & $S I+W M$ & $1.12(0.73,1.75)$ & $1.00(0.67,1.58)$ & $0.66(0.39,1.16)$ & $\underline{0.28(0.22,0.35)}$ \\
\hline & $-3.08(-12.25,5.93)$ & $-3.26(-9.21,3.26)$ & $1.03(-5.62,7.12)$ & SMI + WM & $0.88(0.52,1.51)$ & $0.59(0.31,1.10)$ & $\underline{0.25(0.17,0.36}$ \\
\hline & $-2.60(-12.30,7.90)$ & $-2.69(-10.87,5.50)$ & $1.63(-6.79,9.48)$ & $0.38(-8.25,9.34)$ & SQFZI + WM & $0.66(0.36,1.23)$ & $0.28(0.19,0.39$ \\
\hline & $-3.11(-12.39,6.93)$ & $-3.17(-10.26,4.48)$ & $1.26(-6.88,8.28)$ & $0.25(-8.18,8.53)$ & $-0.50(-10.09,9.22)$ & YQFMI + WM & $0.42(0.25,0.70$ \\
\hline & $4.27(-2.61,11.46)$ & $\underline{4.05(1.00,7.59)}$ & $\underline{8.61(4.22,10.99)}$ & $7.29(1.97,12.70)$ & $6.81(-0.59,14.06)$ & $7.26(0.42,13.64)$ & WM \\
\hline
\end{tabular}

Note: The result underlined meant it had statistical significant

tested in 22 RCTs $[13,19,20,28,30,33,36,38,39,45$, $53,55,58,61,65,72,107,109,111,119,121,122]$ and 8 RCTs $[13,20,30,55,61,107,109,119]$ respectively. According to Table 4, it appeared that there was no significant difference between each comparison.

The ranking analysis suggested that $\mathrm{HQI}+\mathrm{WM}$ and SQFZI+WM was the optimum for these two indexes (Table 5).

\section{ADRs/ADEs}

Among 113 RCTs, a total of 36 [22, 23, 26, 30, 32, 33, 36, $39,40,43,45,48,49,51,54,64,65,67,71-74,77-81,88$, 93, 97-99, 103, 107, 108, 119, 120] RCTs (HQI (2 RCTs), SFI (13 RCTs), SI (13 RCTs), SMI (4 RCTs), SQFZI (2 RCTs), YQFMI (2 RCTs)) did not appear ADRs/ADEs during the trials. Another 72 RCTs (HQI (10 RCTs), SFI (25 RCTs), SI (17 RCTs), SMI (6 RCTs), SQFZI (10 RCTs),

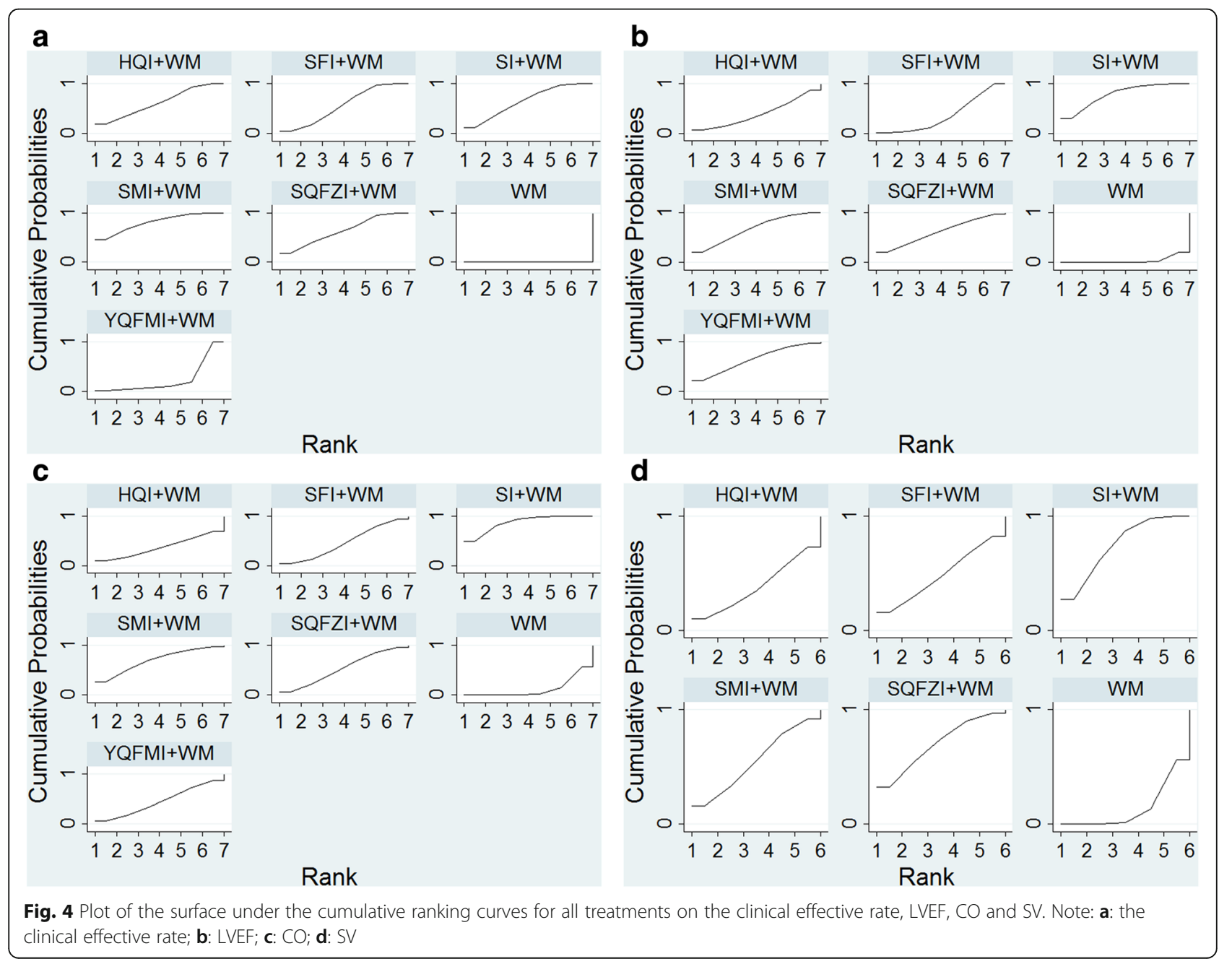


Table 2 Mean difference (95\%Cls) of CO (right upper part) and SV (left lower part)

\begin{tabular}{|c|c|c|c|c|c|c|c|}
\hline \multicolumn{8}{|l|}{$\mathrm{CO}$} \\
\hline \multirow[t]{7}{*}{ sV } & $\mathrm{HQI}+\mathrm{WM}$ & $-0.22(-2.09,1.59)$ & $-0.97(-2.61,0.80)$ & $-0.64(-2.68,1.31)$ & $-0.36(-2.21,1.53)$ & $-0.20(-2.14,1.82)$ & $0.32(-1.29,1.99)$ \\
\hline & $-1.92(-21.24,16.33)$ & $\mathrm{SFI}+\mathrm{WM}$ & $-0.73(-1.69,0.34)$ & $-0.46(-1.89,1.03)$ & $-0.12(-1.36,1.13)$ & $0.03(-1.38,1.49)$ & $0.55(-0.29,1.43)$ \\
\hline & $-6.07(-21.13,9.09)$ & $-4.32(-17.07,9.69)$ & $\mathrm{SI}+\mathrm{WM}$ & $0.29(-1.02,1.51)$ & $0.61(-0.46,1.58)$ & $0.77(-0.54,1.98)$ & $1.29(0.74,1.72)$ \\
\hline & $-3.05(-20.33,14.23)$ & $-1.10(-17.34,15.64)$ & $2.99(-8.76,14.46)$ & SMI + WM & $0.32(-1.15,1.77)$ & $0.46(-1.15,2.13)$ & $0.99(-0.17,2.16)$ \\
\hline & $-5.74(-23.58,11.31)$ & $-3.84(-20.16,12.65)$ & $0.25(-11.63,12.08)$ & $-2.51(-17.27,11.40)$ & SQFZI + WM & $0.14(-1.31,1.65)$ & $0.68(-0.21,1.57)$ \\
\hline & - & - & - & - & - & YQFMI + WM & $0.52(-0.64,1.68)$ \\
\hline & $3.28(-10.90,17.25)$ & $5.12(-6.59,18.01)$ & $\underline{9.35(3.75,14.90)}$ & $6.46(-3.94,16.34)$ & $9.04(-1.36,19.54)$ & - & WM \\
\hline
\end{tabular}

Note: The result underlined meant it had statistical significant

YQFMI (4 RCTs)) did not mention the situation of ADRs/ ADEs. In others RCTs, one of the SFI treatment group occurred 2 cases of mild elevation of blood pressure and 2 cases of slight dry cough, and the corresponding control group occurred 3 cases of slight dry cough and 2 cases of headache [37]. Besides, one of the SI treatment group occurred 2 cases of mild anaphylaxis. There were 3 RCTs with SMI treatment group appeared ADRs [96, 100, 104]. One RCT occurred 1 case of pruritus in the treatment group and 6 cases mild headache in the control group. Another occurred 3 cases of mild gum bleeding in the control group. Another occurred 2 cases of stomach upset in the treatment and control group respectively. All of the symptom were alleviated after corresponding treatment and did not influence the RCTs.

\section{Funnel plot characteristics}

A comparison-adjusted funnel plot for the clinical effective rate was displayed in Fig. 5. The funnel plot was general symmetrical in visual. Thus we concluded that the obvious publication bias did not exist.

\section{Discussion}

The impairment of CHF has been a global public health issue [125], with the utilization of a conjunction between CHIs and WM in its treatment, the efficacy of CHF has been promoted, meanwhile, more and more relevant RCTs and pairwise meta-analysis were carried out. But almost RCTs concerned about the efficacy between a kind of CHI plus WM and WM, many CHIs have not been compared head to head. Thus, researchers could merely figure out the efficacy of a CHI based on these RCTs via pairwise meta-analysis. While NMA can address this void, the efficacy of CHIs can be obtained at a time based on indirect comparison. By comparing with WM, the efficacy of CHIs for CHF and their rank can be demonstrated. we conducted a NMA in order to appraise the efficacy and safety of seven interventions: HQI + WM, SFI + WM, SI + WM, SMI + WM, SQFZI $+\mathrm{WM}, \mathrm{YQFMI}+\mathrm{WM}$ and WM.

This study made an extensive literature review and evaluation. The clinical data derived from 113 RCTs in the aspects of the clinical effective rate, LVEF, CO, SV, $6 \mathrm{MWT}, \mathrm{BNP}$, as well as the value of LVEDD and LVESD. CO, SV, LVEDD and LVESD was regarded as a supplement of cardiac condition, while the consequence of LVEDD and LVESD was no significant difference in most cases, these two outcomes' results were merely deemed as a reference. Besides, 6MWT was vital indicator of patients' recovery, and its importance was emphasized in the guide [9], though the amount of relevant RCTs in this study was small and its statistical power was low, we just treat it as a secondary outcome. In addition, the measurement of BNP was highlighted in guide as an exclusion for CHF [126]. Therefore, we viewed it as a secondary index as well. In terms of the

Table 3 Mean difference (95\%Cls) of 6MWT (right upper part) and BNP (left lower part)

\begin{tabular}{|c|c|c|c|c|c|c|c|}
\hline \multicolumn{8}{|c|}{ UWT } \\
\hline BNP & $\mathrm{HQI}+\mathrm{WM}$ & $-5.40(-71.99,70.70)$ & $-29.10(-115.00,66.32)$ & $16.53(-82.29,163.00)$ & $7.97(-134.30,150.90)$ & - & $17.38(-52.09,84.04)$ \\
\hline & - & $\mathrm{SFI}+\mathrm{WM}$ & $-22.05(-91.18,37.25)$ & $25.48(-84.97,155.60)$ & $15.06(-137.40,161.20)$ & - & $22.42(-9.55,53.97)$ \\
\hline & - & $54.65(-19.88,134.30)$ & $\mathrm{SI}+\mathrm{WM}$ & $50.83(-75.61,178.60)$ & $36.18(-109.80,204.00)$ & - & $45.03(-12.06,101,10)$ \\
\hline & - & $\underline{80.17(16.67,147.5)}$ & $24.53(-58.64,110.20)$ & SMI + WM & $-16.56(-213.70,188.00)$ & - & $-1.23(-133.50,100.10$ \\
\hline & - & $\underline{110.00(35.08,186.40)}$ & $53.69(-41.32,151.3)$ & $27.54(-44.17,108.30)$ & SQFZI + WM & - & - \\
\hline & - & - & - & - & - & YQFMI + WM & $7.12(-141.80,149.50)$ \\
\hline & - & $87.77(32.61,129.90)$ & $34.25(-44.31,100.20)$ & $8.33(-52.43,63.95)$ & $-20.41(-87.19,35.51)$ & - & WM \\
\hline
\end{tabular}

Note: The result underlined meant it had statistical significant 
Table 4 Mean difference (95\%Cls) of LVEDD (right upper part) and LVESD (left lower part)

\begin{tabular}{llllllll}
\hline LVEDD & \multicolumn{7}{l}{} \\
\hline LVESD & HQI + WM & $-6.39(-20.58,8.88)$ & $-5.90(-22.48,11.36)$ & - & $-7.40(-21.99,8.39)$ & $-5.02(-20.71,10.80)$ & $-7.87(-21.64,7.22)$ \\
& $-1.36(-17.43,15.51)$ & SFI + WM & $0.32(-9.47,10.49)$ & - & $-1.04(-6.88,5.10)$ & $1.08(-8.40,10.10)$ & $-1.49(-5.58,2.45)$ \\
& - & - & SI + WM & - & $-1.43(-11.31,8.84)$ & $0.68(-12.36,13.41)$ & $-1.88(-10.71,7.12)$ \\
& - & - & - & SMI + WM & - & - & - \\
& $-2.21(-17.01,13.77)$ & $-0.72(-10.35,8.84)$ & - & - & SQFZI + WM & $2.22(-7.61,11.31)$ & $-0.37(-5.41,3.66)$ \\
& $0.97(-20.42,23.54)$ & $2.30(-15.51,17.43)$ & - & - & $3.02(-14.80,20.13)$ & YQFMI + WM & $-2.68(-10.91,6.07)$ \\
& $-4.03(-18.02,10.96)$ & $-2.57(-10.73,5.24)$ & - & - & $-1.82(-7.68,3.52)$ & $-4.80(-21.19,12.13)$ & WM \\
\hline
\end{tabular}

primary outcomes, SI + WM and SMI + WM exhibited superior performance. What more, these two interventions did a noteworthy effect on $\mathrm{CO}$ and SV. And SI + WM also obtained a first-rank with respect to 6MWT. Overall, on the basis of receiving WM, CHF patients received SI or SMI may be more efficacious. Both of them were approved by CFDA on the market of CHF. SI was derived from Shengmai San which has been widely used for cardiovascular diseases since 1186 in China [127]. It was mainly made from the extractive of Panax ginseng, Radix Ophiopogonis and Schisandra chinensis, and had a function as replenishing qi-yin deficiency. Pharmacological researches have confirmed that SI had features in perfecting cardiac function and alleviating heart failure, enhancing myocardial contractility and cardiac pumping [128]. Under the guideline of TCM, SI was employed in $\mathrm{CHF}$ treatment routinely with its preferable curative effect, and several pairwise meta-analysis manifested that a conjunctive between SI and WM owned a superior capability on increasing the effective rate and LVEF [128-130]. As for SMI, it stemmed from Shenmai Yin which was prescribed by Simiao Sun in the Tang Dynasty [131], and its ingredients did not contain Schisandra chinensis compared with SI, but it also had a superior capacity in nourishing yin and benefiting qi. Upon pharmacological researches, the effect of SMI on promoting myocardial contractility and antiarrhythmic action has been verified [132]. Besides, several pairwise meta-analysis demonstrated that SMI plus WM exhibited a better performance in improving the effective rate, LVEF, CO, SV and decreasing BNP than WM [133-135].

Apart from efficacy, the safety of interventions was the other crucial element that must be considered in clinical trials. In this study, the occurrence rate of ADRs/ADEs was small, but about $64 \%$ of the research did not report the ADRs/ADEs. Hence, we could not draw a certain conclusion on it. As suggested in previous study, anaphylaxis was the main ADRs/ADEs of CHIs, and it would appear within $30 \mathrm{~min}$ at first time [136-139]. Hence, it is crucial for clinicians to monitor the ADRs/ ADEs after using CHIs. Meanwhile, it is necessary to reported exactly if ADRs/ADEs occurred [136].

Upon the design and contents, three merits enhanced the creditable of this study. Firstly, this study made a comprehensive literature search and a contrast for six CHIs which have been already adopted in CHF treatment. Besides, this study expressed the efficacy of CHIs objectively due to the relevant large number of eligible RCTs. Furthermore, a strict eligibility criterion was formulated before implementing NMA. The consistency of the intervention and the curative standard lowered the clinical heterogeneity. What's more, it was significant that the outcomes demonstrated cardiac condition in multiaspect. According to corresponding conclusions, this study provided several clinical suggestions for treatment in CHF.

Table 5 Ranking probability for all treatments on the clinical effective rate, LVEF, CO, SV, 6MWT, BNP, LVEDD and LVESD

\begin{tabular}{|c|c|c|c|c|c|c|c|c|}
\hline \multirow[t]{2}{*}{ Treatment } & \multicolumn{8}{|l|}{ Outcomes } \\
\hline & the clinical effective rate & LVEF & $\mathrm{CO}$ & SV & $6 \mathrm{MWT}$ & BNP & LVEDD & LVESD \\
\hline $\mathrm{HQI}+\mathrm{WM}$ & 0.615 & 0.391 & 0.370 & 0.384 & 0.507 & - & 0.795 & 0.583 \\
\hline $\mathrm{SFI}+\mathrm{WM}$ & 0.559 & 0.359 & 0.462 & 0.481 & 0.596 & 0.020 & 0.503 & 0.533 \\
\hline$S I+W M$ & 0.649 & 0.783 & 0.872 & 0.747 & 0.776 & 0.392 & 0.499 & - \\
\hline$S M I+W M$ & 0.806 & 0.670 & 0.695 & 0.550 & 0.378 & 0.575 & - & - \\
\hline SQFZI+WM & 0.635 & 0.613 & 0.530 & 0.697 & 0.460 & 0.849 & 0.361 & 0.501 \\
\hline YQFMI+WM & 0.236 & 0.650 & 0.449 & - & - & - & 0.563 & 0.619 \\
\hline WM & 0.00 & 0.034 & 0.122 & 0.141 & 0.284 & 0.664 & 0.279 & 0.263 \\
\hline
\end{tabular}




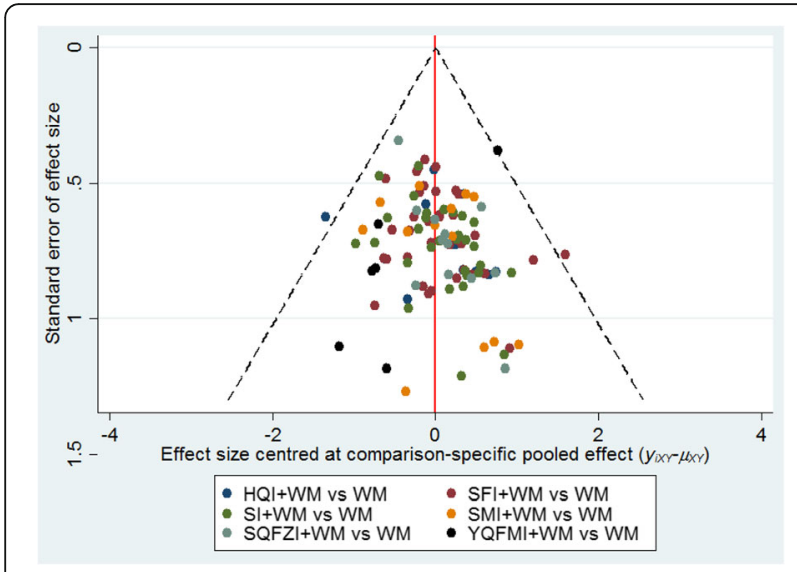

Fig. 5 Funnel plot of the clinical effective rate

\section{Limitation}

Nevertheless, there was still insufficient in this study. Frist, the enrolled patients in RCTs were merely Chinese, which may lead to a bias on whether non-Chinese use eligible CHIs effectively or not. Although CHIs was mostly adopted in China, clinicians also can not only recruit Chinese. Next, just ten of included RCTs reported $6 \mathrm{MWT}$ in this study. While it is 6MWT, and readmission rate that associate with CHF patients closely and influence patients' survival quality. Thus, these aspects should be paid more emphasis when RCTs are designed. In addition, the methodological quality was general, and most included RCTs did not mention the details of randomization and allocation concealment, which may generate an overestimate for eligible CHIs. It should be note that clinicians utilize low risk randomization and concealment method as possible. Based on the limitations, the RCTs conducted in the future should be perfected in relevant areas.

\section{Conclusion}

To sum up, this study found that a combination between SI/SMI and WM exerted a positive effect on improving efficacy of CHF. However, the strength of evidence needed be promoted by more high quality RCTs. Moreover, safety of SI and SMI should be cautious monitoring in trials.

\section{Additional files}

Additional file 1: PRISMA checklist for network meta-analysis. This file contained items about PRISMA checklist for network meta-analysis and corresponding pages of this study. (DOC $66 \mathrm{~kb}$ )

Additional file 2: Search strategy. This file contained the search strategy of traditional Chinese medicine injections and English database. (DOC $39 \mathrm{~kb}$ )

Additional file 3: Characteristics of included randomized controlled trials. This file contained the information about included randomized controlled trials. (DOC $38 \mathrm{~kb}$ )

\section{Abbreviations}

6MWT: 6-min walk test; 95\% Cls: 95\% credible intervals; ACEls: Angiotensinconverting enzyme inhibitors; ADRs/ADEs: Dverse drug reactions/adverse drug events; ARBs: Angiotensin II receptor blockers; BNP: brain Natriuretic peptide; CBM: Chinese Biological Medicine Database; CFDA: China Food and Drug Administration; CHF: Chronic heart failure; CHIs: Chinese herbal injections; CNKI: China National Knowledge Infrastructure; CO: Cardiac output; HQl: Huangqi injection; LVEDD: Left ventricular end-diastolic dimension; LVEF: Left ventricular ejection fraction; LVESD: Left ventricular end-systolic dimension; MD: Mean differences; MeSH: Medical subject headings; NMA: Network meta-analysis; NYHA: New York Heart Association; ORs: Odds ratios; RCTs: Randomized controlled trials; SFI: Shenfu injection; SI: Shengmai injection; SMI: Shenmai injection; SQFZI: Shenqi Fuzheng injection; SUCRA: Surface under the cumulative ranking curve; SV: Stroke volume; TCM: Traditional Chinese medicine; VIP: Chinese Scientific Journal Database; WM: Western medicine; YQFMI: Yiqifumai injection

\section{Funding}

National Natural Science Foundation of China (No. 81473547; No.81673829).

\section{Availability of data and materials}

All data generated or analyzed during this study are included in this published article.

\section{Authors' contributions}

Conception and design of the network meta-analysis: JRW, KHW. Performance of the network meta-analysis: KHW. Quality assessment of the network metaanalysis: MWN, KHW, JRW. Analysis of study data: DZ, XJD. Writing of the paper: $\mathrm{KHW}, \mathrm{DZ}$. All authors read and approved the final version of the manuscript.

\section{Ethics approval and consent to participate}

The ethical approval was not necessary in current meta-analysis because our NMA just gathered the RCTs from literature search, this procedure was without deal with any patients' personal data and harm to any patient.

\section{Consent for publication}

Not applicable.

\section{Competing interests}

The authors declare no competing interests in any aspects.

\section{Publisher's Note}

Springer Nature remains neutral with regard to jurisdictional claims in published maps and institutional affiliations.

Received: 3 October 2017 Accepted: 14 January 2018 Published online: 31 January 2018

References

1. Wang E-W, Jia X-S, Ruan C-W, et al. miR-487b mitigates chronic heart failure through inhibition of the IL-33/ST2 signaling pathway. Oncotarget. 2017;8: 51688-702.

2. Zhao Z-Q, Mao J-Y, Wang X-L, et al. Application and evaluation of Chinese medicine in treatment of chronic heart failure. Chin J Integ Med. 2013;33:1701.

3. Wu D-H. Effects of the shenfu injection on the level of serum cerebral sodium peptide in patients with chronic heart failure. Zhejiang J Integ Tradit Chin West Med. 2013;23:837-8.

4. Vetrovsky T, Siranec M, Parenica J, et al. Effect of a 6-month pedometerbased walking intervention on functional capacity in patients with chronic heart failure with reduced (HFrEF) and with preserved (HFpEF) ejection fraction: study protocol for two multicenter randomized controlled trials. J Transl Med. 2017;15:153.

5. Wang Y-Z, Zhuang Y, Gu Y, Chen Q-Q, Wang Z-X. Progress in the evolution of chronic heart failure syndrome. Guiding J Tradit Chin Med Pharmacol. 2016;22:62-8.

6. Sane R, Aklujkar A, Patil A, et al. Effect of heart failure reversal treatment as add-on therapy in patients with chronic heart failure: a randomized open-label study. Indian Heart J. 2017:69:299.

7. Leucht S, Cipriani A, Spineli L, et al. Comparative efficacy and tolerability of 15 antipsychotic drugs in schizophrenia: a multiple-treatments meta-analysis. Lancet. 2013;382:951-62. 
8. Hutton B, Salanti G, Caldwell DM, et al. The PRISMA extension statement for reporting of systematic reviews incorporating network meta-analyses of health care interventions: checklist and explanations. Ann Intern Med. 2015; 162:777-84.

9. Cardiology Branch of the Chinese Medical Association. Guidelines for the diagnosis and treatment of heart failure in China 2014. Chin J Cardiol. 2014; 42:98-122.

10. Zheng Y-Y. Clinical guideline of new drugs for traditional Chinese medicine. Beijing: Chin Med Sci and Tech Press; 2013. p. 77-85.

11. Zhang $D$, Wu J-R, Liu S, Zhang X-M, Zhang B. Network meta-analysis of Chinese herbal injections combined with the chemotherapy for the treatment of pancreatic cancer. Med. 2017;96:e7005.

12. Chang L, Guo R. Comparison of the efficacy among multiple chemotherapeutic interventions combined with radiation therapy for patients with cervix cancer after surgery: a network meta-analysis. Oncotarget. 2017:8:49515-33.

13. Xing G-P, Nie X-M. Effects of astragalus injection on hemodynamics in patients with congestive heart failure. Med ind inf. 2006;3:67-9.

14. Gu X, Shang S-Z, Guo R. 68 cases of congestive heart failure assisted by astragalus injection. Her Med. 2003;22:556-7.

15. Zhang Z-B. Clinical observation of astragalus injection combined with conventional western medicine for senile patients of chronic congestive heart failure. J New Chin Med. 2017:49:25-8.

16. Luo X-C. Clinical observation of 30 cases of congestive heart failure treated by huangqi injection. J Emerg Syndromes Tradit Chin Med. 2003;12:35.

17. Feng L-Y, Hao W. Clinical observation of 34 cases of congestive heart failure treated with astragalus injection. Chin I Integr Med Cardio/ Cerebrovasc Dis. 2008;6:1362-3

18. Gao J-H. Clinical observation of astragalus injection for treating congestive heart failure. Acad J Guangdong Coll Pharm. 2005;21:610-1.

19. Yan F-Y, Lin H-Z. 80 cases of chronic congestive heart failure treated with astragalus injection. Jiangxi J Tradit Chin Med. 2009;40:27-8.

20. Lin Y, Huang C-L, Zhu C-L. Clinical study on astragalus injection in treating chronic congestive heart failure. Chin Pharm. 2009;18:22-3.

21. Zhang C-M, Duan Q-F, Bian X-J. Clinical observation of astragalus injection in the treatment of chronic heart failure. Med Front. 2013;17:19-20.

22. Yuan G-P. Evaluation of clinical efficacy of astragalus injection for treatment of chronic heart failure. Chin J Clin Pharmacol Ther. 2003;8:710-1.

23. Wang $X-X$. Treatment of chronic heart failure by astragalus injection. Chin Foreign Health Abstr. 2015;14:53-4.

24. Jia B-Q, Xu L. Effect of astragalus injection on chronic heart failure. Chin J Clin Res. 2013;26:654-6.

25. Wang X-G. Effects of shenfu injection on chronic heart failure induced by different etiology and the effect of b-type natriuretic peptide. Speci health 2014;4:598.

26. Wang $\mathrm{H}$, Hu Y-H, Song Q-Q, Qiu Z-L, Bo R-Q. The impact of shenfu injection on the immune function in patients with chronic heart failure and heart kidney yang deficiency syndrome. Chin I Integr Med Cardio/ Cerebrovasc Dis. 2016;14:1441-6.

27. Zhou K, Lin S-Y, Yang WM. Effects of injection on NT-proBNP in heart failure patients with coronary heart disease. J Emerg Syndromes Tradit Chin Med. 2013;22:1625-6.

28. Xu L-L, Guan C-Y, Wang C-Y, Chen J, Zhang L-L. Effects of shenfu injection on the nt-probnp and life quality of chronic heart failure patients. J Emerg Syndromes Tradit Chin Med. 2015;24:918-20.

29. Cui $Y$, et al. Effect of shenfu injection on cardiac function and blood index in patients with chronic heart failure. Hebei med. 2016;22:1623-5.

30. Wu H-Q. Study about efficacy of shenfu injection on cardiac function and bone marrow stem cell mobilization in cardiac failure. Guang'anmen hospital. 2008;

31. Chen G-Q, Wu H-Q, Chen G-L, Dong L-M, Wang Z-M. Effect of shenfu injection on the serum NT-proBNP level of patients with heart failure. J Emerg Syndromes Tradit Chin Med. 2014;24:134-5.

32. Ren $\mathrm{Q}$, Wang $\mathrm{Q}$, Lin $\mathrm{P}$. Clinical observation of the effect of shenfu injection on chronic heart failure. Zhejiang J Integr Tradit Chin West Med. 2015;25:350-2.

33. Cheng $\mathrm{G}$, et al. Observation of clinical efficacy of shenfu injection in patients with congestive heart failure. Shaanxi Med J. 2014;43:482-4.

34. Li L-N. Observation of clinical efficacy of shenfu injection in treating congestive heart failure. J Emerg Syndr Tradit Chin Med. 2016;25:2375-7.

35. Mao X-Z. Clinical effect of shenfu injection on patients with chronic heart failure. J Baotou Med Coll. 2016;32:92-3.
36. Wu H-J, Duan H-W. Clinical study of shenfu injection for heart failure of coronary heart disease. Chin J Integr Med Cardio/ Cerebrovasc Dis. 2009;7:505-7.

37. Li C-Y. Observation of the effect of shenfu injection on chronic heart failure in elderly patients. Academic Conference of Gansu Province Medical Association; 2012

38. LV H-Y. Effects of shenfu injection on treatment of chronic heart failure and its effect on plasma BNP. Liaoning J Tradit Chin Med. 2014;41:726-7.

39. Yang Z-Y, Dong J-Y, Miao L-H. Clinical observation of shenfu injection in elderly patients with chronic heart failure. J Emerg Syndr Tradit Chin Med. 2010;19:2058-9.

40. Cao Z-Q. Observation of efficacy of shenfu injection in treating congestive heart failure in elderly patients. Chin Foreign Med Treat. 2014;30:118-9.

41. Bao G-H, Yu L-H. 30 cases of chronic congestive heart failure were treated with shenfu injection. Chin Med Mod Dis Educ China. 2011;9:27.

42. Zhang A-P, Song G-P, Cai J-S. Clinical observation of 40 cases of chronic congestive heart failure treated with shenfu injection. J Emerg Syndromes Tradit Chin Med. 2011;20:1140-1.

43. Liu Y. Clinical observation of 40 cases of chronic congestive heart failure treated with shenfu injection. J Emerg Syndromes Tradit Chin Med. 2008;16:51-2.

44. Ma L-P. Clinical study on 98 cases of chronic congestive heart failure treated with shenfu injection. World Health Dig. 2013;9:117.

45. Yao J, Lu X-R. Clinical observation on the treatment of chronic congestive heart failure with shenfu injection. J Med Theory Pract. 2010;23:287-8.

46. Ran X, Chen L, Wu W. Clinical observation of shenfu injection for chronic congestive heart failure. J New Chin Med. 2012;44:15-7.

47. Ding L. Observation of the effect of shenfu injection on chronic heart failure. Clin J Tradit Chin Med. 2013;25:482-3.

48. Zhu G-Y. Clinical observation of shenfu injection in the treatment of chronic heart failure. Hubei University of Traditional Chinese Medicine. 2013;

49. Qiu Q. Clinical observation of shenfu injection in treating chronic heart failure. Hunan J Tradit Chin Med. 2013;29:33-5.

50. Wang C-L. Clinical observation of 100 cases of chronic heart failure treated with shenfu injection. J Emerg Syndromes Tradit Chin Med. 2012;29:958-9.

51. Liu D-H, Chen G-Y, Chen W-X. Clinical observation of the curative effect of shenfu injection on chronic heart failure. The fourth Traditional Chinese Medicine continued education peak conference. 2011;

52. Qiu W-W. Clinical observation of the effectiveness of shenfu injection on chronic heart failure. J Emerg Syndromes Tradit Chin Med. 2010;19:420-1.

53. Qin Y, Zhou Q, Chen Y, Li J-P, Lv X-F. Clinical observation on the treatment of chronic heart failure with shenfu injection. J Emerg Syndromes Tradit Chin Med. 2015:24:161-2.

54. Fan $T-B$, Yang Z-X. Clinical observation on the treatment of chronic heart failure with shenfu injection. J Emerg Syndromes Tradit Chin Med. 2012;21:1851-2.

55. Fu R. Clinical observation on the treatment of chronic heart failure with shenfu injection. J Chin Tradit Chin Med Inf. 2011;3:245-6.

56. Jin Z-C, Jin Z-X, Han L. Study on clinical efficacy of shenfu injection in the treatment of chronic heart failure. Pharmacol Clin Chin Mater Med. 2015;31:159-61.

57. Gao F. Effect of shenfu injection on inflammatory cytokines in patients with chronic heart failure. J Emerg Syndromes Tradit Chin Med. 2012;21:1646-7.

58. Wang J, Zhang J, Ran G-Y. Clinical effect of shenfu injection on chronic heart failure and its impact on serum hs-CRP and IL-6. J Hubei Univ Chin Med. 2015;17:10-2.

59. Jiang L-X, Luo P. 96 cases of heart failure in treatment with shenfu injection. Shaanxi J Tradit Chin Med. 2011;32:1285-6.

60. Zhang Z-F, Lv H-G. Observation on the curative effect of shenfu injection on chronic heart failure in elderly patients. People's Mil Surg. 2009;52:360-1.

61. Liu D-Q, Zheng Z-M, Zhang K. Treatment of chronic congestive heart failure with shenfu injection. Jiangxi J Med Pharm. 2005;40:529-30.

62. Yu H-B, et al. Changes of nitric oxide synthase, adrenal medulla lignin, interleukin-10 in heart failure patients and the intervention effect of shenfu injection. Chin J Gerontol. 2014;34:7085-6.

63. Wang Y-X. Clinical observation on the short-term treatment of chronic congestive heart failure in elderly patients with shengmai injection. Chin Tradit Pat Med. 2010;32:713-4.

64. Wang G-T, He Y-Q, Yang P. Effects of shengmai injection on cardiac function and plasma brain natriuretic peptide in patients with congestive heart failure. Chin J Integr Med. 2010;30:551-3.

65. Zhou J. Clinical effect of shengmai injection on plasma BNP in patients with congestive heart failure. J Emerg Syndromes Tradit Chin Med. 2009;18:732-3. 
66. Pan J-J. Therapeutic effect of shengmai injection on chronic heart failure. China Contemp Med. 2014;20:70-1.

67. Wang Z-Y, Hu X-Y, Liu F-Z. Clinical observation of shengmai injection for congestive heart failure. Lishizhen Med Mater Med Res. 2004;15:353.

68. Zhao Q-F, Dai J, Bai X-L. Clinical efficacy of shengmai injection as adjuvant therapy for elderly patients with chronic congestive heart failure: an analysis of 31 cases. Hunan J Tradit Chin Med. 2015;31:23-5.

69. Wu C-Z, Hu M. Clinical observation of 36 cases of chronic congestive heart failure in elderly patients. Yunnan J Tradit Chin Med Mater Med. 2011;32:30.

70. Yang Z-Z, Zhang Z. Clinical observation of 43 cases of chronic congestive heart failure assisted by shengmai injection. Chin Med Her. 2010;7:80-1.

71. Cheng F-Y. Clinial treatment of 45 cases of chronic congestive heart failure with shengmai injection. Chin J Integr Med Cardio/ Cerebrovasc Dis. 2007;5: 1239-40.

72. Ni Y-M. 43 cases of chronic heart failure in treatment with shengmai injection. Her Med. 2012;31:171-2.

73. Xu J. 50 cases of chronic heart failure in treatment with shengmai injection. J M Udanjiang M Ed Coll. 2004;25:32-3.

74. Wen Y. Shengmai injection combined with conventional drug in the treatment of chronic heart failure for 20 cases. Chin Med Mod Dis Educ China. 2016;14:139-41.

75. Wen Y. Efficacy of shengmai injection in treatment of 64 cases with chronic heart failure. J Chengdu Univ Tradit Chin Med. 2013;36:76-8.

76. Kong LG, Zhu KW. Clinical observation of 30 cases of congestive heart failure in treatment of congestive heart failure. J Clin Emerg Call. 2004:5:26-7.

77. Tang E-W, Zeng Y-R. Observation of 33 cases of congestive heart failure treated by shengmai injection. Youjiang Med J. 2011;29:10-1.

78. Li W-D, Zhang T-S. Therapeutic effect of shengmai injection on congestive heart failure. Chin Commun Doct. 2002;18:35.

79. Chen Z-G. Therapeutic effect of shengmai injection on chronic heart failure in elderly patients. For All Health. 2016;10:28.

80. Luo $X-Y$. Therapeutic effect of shengmai injection on chronic heart failure in elderly patients. Med Inf. 2014;27:271.

81. Zhai Y-M. Clinical research of pulse-activating injection on 30 cases of chronic congestive heart failure. J Henan Univ Chin Med. 2009;24:59-61.

82. He D-Y, Wang P, Liu Q. Clinial treatment of 20 cases of chronic heart failure with shengmai injection. Henan Tradi Chin Med. 2006;26:71-2.

83. Chen $\mathrm{K}-\mathrm{H}, \mathrm{Xu} \mathrm{Z}-\mathrm{Q}$, Liu QS. Analysis of 36 cases of chronic heart failure treated by shengmai injection. Zhejiang J Integr Tradit Chin West Med. 2008;18:155-6.

84. Wu X. Clinical observation of 52 cases of chronic heart failure treated by shengmai injection. For All Health. 2013;7:56.

85. Lu F. Clinical observation of 68 cases of chronic heart failure treated by shengmai injection. J Emerg Syndromes Tradit Chin Med. 2012;21:1695.

86. Liu S-H. Clinical observation of 120 cases of chronic heart failure treated by shengmai injection. National academic seminar on Wang qingren thought. 2008;

87. Zou X, Shi S-Q, Han Y. Clinical observation of the treatment of chronic heart failure by shengmai injection. Clin J Tradit Chin Med. 2011;23:777-8.

88. Kong W-W. Study on shengmai injeetion in patients with chronic heart failure of type qi and yin deficiency. Guangdong University Traditional Chinese Medicine; 2010.

89. Ni L-M, Ding C-Y. Curative effect observation on the treatment of congestive heart failure. Zhejiang J Tradit Chin Med. 2007:42:668.

90. Wang R-L, Zhou X-Y, Tian X-Z. 80 cases of chronic heart failure in treated with shengmai injection. Med Inf. 2007;20:326-7.

91. Li S-G. Clinical study on effects of shengmai injection with chronic heart failure treatment by monitoring the level of plasma NT-probnp. Fujian College of Traditional Chinese Medicine. 2009;

92. Zhang Y-T. Clinical effect of Chinese and western medicine on 103 cases of chronic congestive heart failure. Xinjiang J Tradit Chin Med. 2015;33:42-3.

93. Shi G-R. Treatment of chronic heart failure by Chinese and western medicine. Liaoning University of Traditional Chinese Medicine. 2009;

94. Pan Q-H, Chu S-X, Zheng Z-X, Jiang L-Q, Chen J-S. Effect of shenmai injection on the efficacy and plasma BNP of chronic congestive heart failure. Zhejiang J Tradit Chin Med. 2014;49:422.

95. Huang S-E, Huang Q, Yao Q, Pei D-A. Influence of shenmai injection on chronic contractive heart failure patients' heart function and BNP. J Zhejiang Univ Tradit Chin Med. 2011;35:718-9.

96. Hou X-L, Hong J-K, Xiao X-Y, Chen S-X. Effect of shenmai injection on cardiac function and brain natriuretic peptide in patients with chronic heart failure. Mod J Integ Tradit Chin West Med. 2014;23:2904-5.
97. Wang J-L, Zhou W-J, Shi G-P. Clinical observation of 51 cases of senile heart failure assisted by shenmai injection. Suzhou Univ J Med Sci. 2010; 30:1289-90.

98. Liu XR. Clinical study of combined western medicine with shenmai injection to treat chronic heart failure. World Health Dig Med Period. 2011;8:103.

99. Qu F. Clinical observation of shenmai injection to treat congestive heart failure. J Emerg Syndromes Tradit Chin Med. 2006;15:1102.

100. Wu QG. Effect of shenmai injection on chronic congestive heart failure. Mod J Integr Tradit Chin West Med. 2011;20:3961-2.

101. Tian J-H, Zhang R-H. Clinical efficacy of shenmai injection in chronic heart failure. Chin Med Mod Dis Educ China. 2010;8:98-9.

102. Cui H-S. Analysis of 42 cases of chronic heart failure treated by shenmai injection. Neimonggu J Tradit Chin Med. 2008;27:65-6.

103. Hu C-L. Effective observation on shenmai injection for chronic heart failure of 132 cases. Med J West China. 2011;23:2162-3.

104. Ye J-F. Effect of shenmai injection on the treatment of chronic heart failure and its effect on plasma cerebral natriuretic peptide. Mod Pract Med. 2015; 27:201-2.

105. Wang J. Therapeutic effective observation on 22 cases of congestive heartfailure treated with combined Chinese traditional and western therapy. Hunan J T Radit Chin Med. 2002:18:7-8.

106. Guo H-J, Tao Q-X, Li S-L. Chinese and western medicine combined treatment of 58 cases of chronic heart failure. Jilin J Tradit Chin Med. 2012;32:681-3.

107. Wu Y. Effect of shenqi fuzheng injection on the left ventricle of the heart and BNP on patients with chronic heart failure. Pract J Cardiac Cereb Pneum Vasc Dis. 2014;22:45-6.

108. Su H-M. Observation of the quality of chronic ischemic slow heart failure in the injection of shenqi fuzheng injection. Chin Commun Doct. 2009;11:163-4.

109. Wang L-W. Observation of shenqi fuzheng injection on cardiac function, BNP and myocardial troponin in patients with chronic congestive heart failure. Mod J Integ Tradit Chin West Med. 2014;23:1766-8.

110. Wang $L$, Ye B-H, Wang D-X, Li BT. Effects of shenqi fuzheng injection on plasma $n$ terminal pro-brain natriuretic peptide in patients with chronic heart failure. Pract J Cardiac Cereb Pneum Vasc Dis. 2011;19:1774-5.

111. Liu $P, X u X-Y, L i ~ N$. Effect of shenqi fuzheng injection on peripheral blood ANGII, ET-1 levels and clinical curative effect in patients with congestive heart failure. Chin J Biochem Pharm. 2015;35:81-3.

112. Liang S-X. Observation of 43 cases of chronic congestive heart failure with elderly patients in treating with shenqi fuzheng injection. J New Chin Med. 2009;47:74-5.

113. Chen F-J, Chen L-C. Clinical curative effect and safety of shenqi fuzheng injection in the treatment of elderly patients with chronic heart failure. Chin J Biochem Pharm. 2017:37:137-9.

114. Yang S-J. Observation of curative effect of shenqi fuzheng injection on chronic congestive heart failure. World Latest Med Inf. 2015;15:166-7.

115. He F-T. Observation of curative effect of shenqi fuzheng injection on congestive heart failure. J Emerg Syndromes Tradit Chin Med. 2003;12:399-40.

116. Yun M-L. Clinic observation of 55 cases with chronic heart failure treated by shenqi fuzheng injection. Nat Sci J Hainan Univ. 2006;24:371-3.

117. Wu Z-G, Feng F-J, Xie W-B. Clinical effect of shenqi fuzheng injection on the treatment of chronic heart failure. Res Integrated Tradit West Med. 2015;7:236-7

118. Mao H-Y. Clinical observation of chinese and western medicine in treating chronic congestive heart failure. J Pract Tradit Chin Med. 2014;30:120-1.

119. Wang H-P, Wu Z-G. Clinical observation of digoxin tablet combined with yiqifumai in treatment of chronic heart failure. Drugs \& Clin. 2014;29:532-5.

120. Zhao Y-B. Clinical observation on treating chronic heart failure by yiqifumai injection. Mod Med J China. 2015;17:72-3.

121. Xue $L-X$, Wang $H-L$, Lei $X$, Feng $L$. Effects of yiqifumai injection on cardiac function and plasma BNP in chronic heart failure. Chin J Integr Med Cardio/ Cerebrovasc Dis. 2014;12:279-80.

122. Ren $\mathrm{H}$. Clinical observation on treating chronic heart failure by yiqifumai injection (freeze-dried) combined with trimetazidine. The World Clin Med. 2016;10:4-5

123. Yang C-L, Liu Z-H. Clinical study on the treatment of chronic heart failure in elderly patients with coronary heart disease by yiqifumai injection (freeze-dried). Pract Geriatr. 2014;28:607-8

124. Zhao X-F, Liu J-Y, Zhang M-L. Clinical efficacy of injection therapy for chronic heart failure with yiqifumai injection (freeze-dried). Shanxi Med J. 2015;44:1533-5.

125. Punchik B, et al. Can home care for homebound patients with chronic heart failure reduce hospitalizations and costs? PLoS One. 2017;12:e0182148. 
126. Cui W. Highlights of european society of cardiology guidelines for the diagnosis and treatment of acute and chronic heart failure 2012. Chin J Cardiovasc Med. 2012;16:324-6.

127. Chen $C-Y$, Lu L-Y, Chen $P$, et al. Shengmai injection, a traditional Chinese patent medicine, for intradialytic hypotension: a systematic review and meta-analysis. Evid Based Complement Alternat Med. 2013;2013:1-14.

128. Chen J, Luo M-X, Zheng Q, Zhou Y-C. Effect of shengmai injection on patients with chronic heart failure: a meta-analysis. Chin J Inf Tradit Chin Med. 2011;18:25-9.

129. Hu Z-Z, Tang L-M, Lin Y. Meta-analysis of the efficacy of shengmai injection in treating congestive heart failure. Zhejiang J Integr Tradit Chin West Med. 2016;26:32-7.

130. Yuan Y, Mao J-Y, Tang E, Hou Y-Z, Wang X-L. Treatment of chronic heart failure with western drugs combining shengmai injection: a systematic review on randomized controlled trials. Chin J Evid Based Cardiovasc Med. 2014;6:519-24

131. Shi L, Xie Y, Liao X, Luo Y. Shenmai injection as an adjuvant treatment for chronic cor pulmonale heart failure: a systematic review and meta-analysis of randomized controlled trials. BMC Complement Altern Med. 2015;15:1.

132. Duan Y-P, Meng C-L, Li P. Pharmacological action and clinical application of shenmai injection. Chin Med Abstr. 2007;23:698-700.

133. Chen H-D, Xie Y-M, Wang L-X, Wu J-B. Systematic review of efficacy and safety of shenmai injection for chronic heart failure. China J Chin Mater Med. 2014;39:3650-62.

134. Hou Y-Z, Mao J-Y, Wang X-L, Liu C-X, Zhang C. Shenmai injection in heart failure patients: a systematic review and meta-analysis. Chin J Evid Base Med. 2010;10:939-45.

135. Duan P. Systematic review of efficacy and safety of shenmai injection in treatment of heart failure. J Taishan Med Coll. 2014;35:1063-4.

136. Yan W-L, Tong X-T, Yang S-Q. The causes and countermeasures of adverse reactions of traditional Chinese medicine injection. Mod Tradit Chin Med. 2016:36:56-8.

137. Liu J-G, Liu Y-L, Zhang H-M, Li Y-L. Exploration on the adverse reactions of chinese herbal injection and the preventive measures. World J Integr Tradit West Med. 2017;12:81-4.

138. Hao C, Liu F-Q. Progress on cause of adverse reaction of traditional Chinese medicine. J Pharm Res. 2017;36:369-72.

139. Zhang X-X. A review on adverse reaction from TCM injection. Clin J Chin Med. 2017;9:141-2.

\section{Submit your next manuscript to BioMed Central and we will help you at every step:}

- We accept pre-submission inquiries

- Our selector tool helps you to find the most relevant journal

- We provide round the clock customer support

- Convenient online submission

- Thorough peer review

- Inclusion in PubMed and all major indexing services

- Maximum visibility for your research

Submit your manuscript at www.biomedcentral.com/submit

) Biomed Central 